\title{
Anomalous diffraction of matter waves with minimal quantum metasurfaces
}

\author{
Constantinos Valagiannopoulos ${ }^{1 *}$ (D)
}

"Correspondence:

valagiannopoulos@gmail.com

1 Department of Physics, Nazarbayev

University, Nur-Sultan, Kazakhstan

\begin{abstract}
In the last few years, there is a huge upsurge in the number of closed deals regarding quantum technologies for materials, computing, communication and instrumentation. Such a trend has inevitably affected the research funding market; thus, large state initiatives are taken that are directly expected to drive the formulation of novel research concepts and the development of quantum device prototypes from sensors and circuitry to quantum memory and repeaters. A fundamental operation behind all these applications is the effective steering of electrons, constituting matter waves, along specific directions and with certain magnitudes, due to development of various reflective and refractive orders. The objective of this study is to optimize the simplest structure that supports such anomalous diffraction, namely a quantum metasurface comprising cylindrical rods embedded in suitable crystalline matter. Several highly-performing designs from these minimal setups are proven to work exceptionally as multiport components, employable to a variety of quantum engineering implementations.
\end{abstract}

Keywords: Quantum metasurface; Anomalous diffraction; Matter waves

\section{Introduction}

Quantum technologies are today developing at a rapid pace and providing novel concepts that address in a revolutionary manner multiple core scientific problems. Quite high on this list one may find quantum sensing that boosts dramatically the optical resolution of extremely close point-like sources [1] and sets new limits in spectroscopy and metrology via quantum interpolation [2]. Such evolutions have led to demonstration of quantum memory that enhances the interval over which phases are accumulated, beyond the coherence lifetime [3]. Moreover, quantum communication technologies based on solid-state devices enabling stogage of quantum entanglement have been proposed, paving the way for building multiplexed repeaters for long-distance quantum networks [4]. Importantly, whole classes of computational tasks have been found to be executed exponentially faster on a quantum processor than on a classical one, regardless of the employed algorithm, indicating a first realization of quantum supremacy [5, 6]. Even quantum network architectures have become feasible via interconnections that convert quantum states from one physical system to those of another in a reversible way [7].

(c) The Author(s) 2021. This article is licensed under a Creative Commons Attribution 4.0 International License, which permits use sharing, adaptation, distribution and reproduction in any medium or format, as long as you give appropriate credit to the original author(s) and the source, provide a link to the Creative Commons licence, and indicate if changes were made. The images or other third party material in this article are included in the article's Creative Commons licence, unless indicated otherwise in a credit line to the material. If material is not included in the article's Creative Commons licence and your intended use is not permitted by statutory regulation or exceeds the permitted use, you will need to obtain permission directly from the copyright holder. To view a copy of this licence, visit http://creativecommons.org/licenses/by/4.0/. 
All the aforementioned scientific breakthroughs leading to modules most of which are at an infant stage, have ignited large quantum research investments from industry titans and prioritized quantum information science in careful strategic plans [8, 9]. In particular, several workshops on the future of quantum engineering as an emerging branch [10] and brainstorming in roundtables on how the research competitiveness will be maintained in quantum computing [11], culminated in impressive initiatives [12]. Such huge bills are expected to further stimulate research on quantum technologies in the near future even though the funding interest is already high. Only the last few years, several MultiUniversity Research Initiatives (MURIs) have been approved from Army Research Office (ARO) on ab-initio characterization of quantum materials [13], from Air Force Office of Scientific Research (AFOSR) on certifying untrusted quantum devices [14] and from US Department of Defense (DoD) on quantum state control of molecular collision dynamics [15], all concerning the suitable pairing of matter waves with quantum texture.

A backbone operation behind all these fancy applications is the steering of quantum particle beams along specific routes and with certain intensities to be processed accordingly for computation, simulation or memory purposes. It is usually achieved by crafting sophisticated paths of specific material on a host base, along which the quantum signals are guided [16] or exploiting spin-orbit interaction into ring structures to make them work as splitters [17]. Similarly, quantum interference is used for spectral demultiplexing with help from directional couplers and add-drop filters, all these integrated on a single monolithic silicon chip [18]. The same on-chip philosophy is adopted by solid-state quantum sensors with applications in magnetometry, thermometry and chemical sensing [19]. Moreover, a superconducting quantum processor with multiple transmon qubits coupled via coplanar waveguide resonators has been proposed [20] while photonics quantum circuits enabling generation, manipulation, and analysis of multidimensional quantum systems have been experimentally tested [21].

One may accomplish efficient quantum signal steering alternative to opening guidance paths into a background matrix [22], by developing a structure that diffracts the incoming matter waves at controllable axes and pre-determined probabilities. Such an idea is borrowed from Photonics, where suitable phase discontinuities to reflective or refractive waves are imprinted through metasurfaces of optical resonators [23] to develop anomalous diffractive phenomena that, in turn, formulate multi-port networks with tailorable their overall response [24]. Anomalous effects, namely diffraction along counter-intuitive directions, has been investigated in Quantum Mechanics but only in the context of measuring the response for guessing the inhomogeneities size/shape into a host matrix; it aims at recovering non-destructively the composition of embedded nanostructures and makes a hard three-dimensional inverse problem $[25,26]$. However, deliberately creating simplyshaped inclusions, like cylindrical posts, into a background medium is a relatively feasible task via several fabrication methods. In particular, one can engrave chemically nanowires [27] or epitaxially grow radially inhomogeneous elongated heterostructures [28], either following a bottom-up building [29] or top-down dipping [30] approach.

In this work, we investigate the simplest version of this realistically constructable class of quantum devices, namely a periodic metasurface comprising circular cylindrical cavities of specific texture and large length, being embedded into a background host [31]. The structure is obliquely illuminated by a beam of electrons and explicit expressions for the directions and the magnitudes of the developed diffraction waves are obtained. 
The analyzed effect is based on the interference of electron beams whose principles have been formulated many decades ago [32]. However, our work differs from studies of similar objectives that use multiple gratings but do not solve analytically the boundary value problems [33, 34] or employ isolated rods without collective operations [35]. Particular emphasis is placed on the first anomalous order and various combinations of inclusion materials and backgrounds are examined; for each of them, the incidence angle, the energy of the incoming beam and the cylinders size are selected so that the anomalous reflection or transmission is maximized. It is found that quantum well configurations give higher scores than their quantum barrier counterparts [36] while the operation of the device as a quantum multiport with several diffractive channels, is demonstrated. Therefore, this study gives numerous alternative minimal designs for splitting and steering components that may be utilized in various quantum setups from circuitry and memory elements to signal processing and repeaters.

\section{Mathematical formulation}

\subsection{Configuration and assumptions}

Let us consider a plane matter wave created by an electron beam whose particles move along a specific direction with equal probability of existence across the planes normal to it. The beam is traveling into a host medium whose macroscopic potential energy is denoted by $V_{0}$ while the effective mass of an electron within equals $m_{0}$; since the developed matter wave is propagating, the kinetic energy of its particles is larger (by $E$ ) than the local potential level $V_{0}$. It should be declared that, in this model, the impurity of phonon scattering is not taken into account and, accordingly, the electron wave is regarded as coherent [37]; in other words, the particle beam can be represented by a specific wave vector. Therefore, the incoming beam is characterized by a single energy $E$ which is feasible if passed through an energy-selective setup like tilted nanocavities [38] or planar layers [39]. The impinging quantum wave meets obliquely (under angle $\theta$, as in Fig. 1) a grating with period $2 b$ comprising cylindrical inclusions of radius $a<b$ and filled with a different quantum material of energy $V=V_{0}-\Delta V$ and effective mass $m$.

From fundamentals of Quantum Mechanics [40, pp. 11-15], we know that, the key quantity determining the interaction of any e-beam with its environment is the wave function $\Psi(\mathbf{r})$ whose squared magnitude is proportional to the probability of finding the electron at a point with position vector $\mathbf{r}$. The time-independent Schrödinger equation [41, p. 31] respected by the wave function of any e-beam is written as:

$$
\nabla \cdot\left[\frac{1}{m(\mathbf{r})} \nabla\right] \Psi(\mathbf{r})+\frac{E-V(\mathbf{r})}{\hbar^{2} / 2} \Psi(\mathbf{r})=0,
$$

where $E$ is the total energy of the beam, $V(\mathbf{r})$ the local potential energy and $m(\mathbf{r})$ the local effective mass; obviously $\hbar$ stands for the reduced Planck constant.

Note that in Fig. 1, we assign to each of the cylindrical inclusions an integer number $n$ and we define infinite coordinate systems $\left(r_{n}, \varphi_{n}\right) \equiv\left(x_{n}, y_{n}\right)$ for $n \in \mathbb{Z}$, each one centralized at one post. The global coordinate system $(r, \varphi) \equiv(x, y)$ is assumed the one with $n=0$. The wave function describing the incoming matter wave, if the parallel wires were absent, takes the form $\Psi_{\text {back }}=e^{-i k_{0} x \cos \theta-i k_{0} y \sin \theta}$, where $k_{0}=\sqrt{2 m_{0} E} / \hbar$ is the wavenumber of the impinging beam into background medium. On the contrary, by adopting partial wave formalism [42, p. 596], the wave function into the central $(n=0)$ post is written 


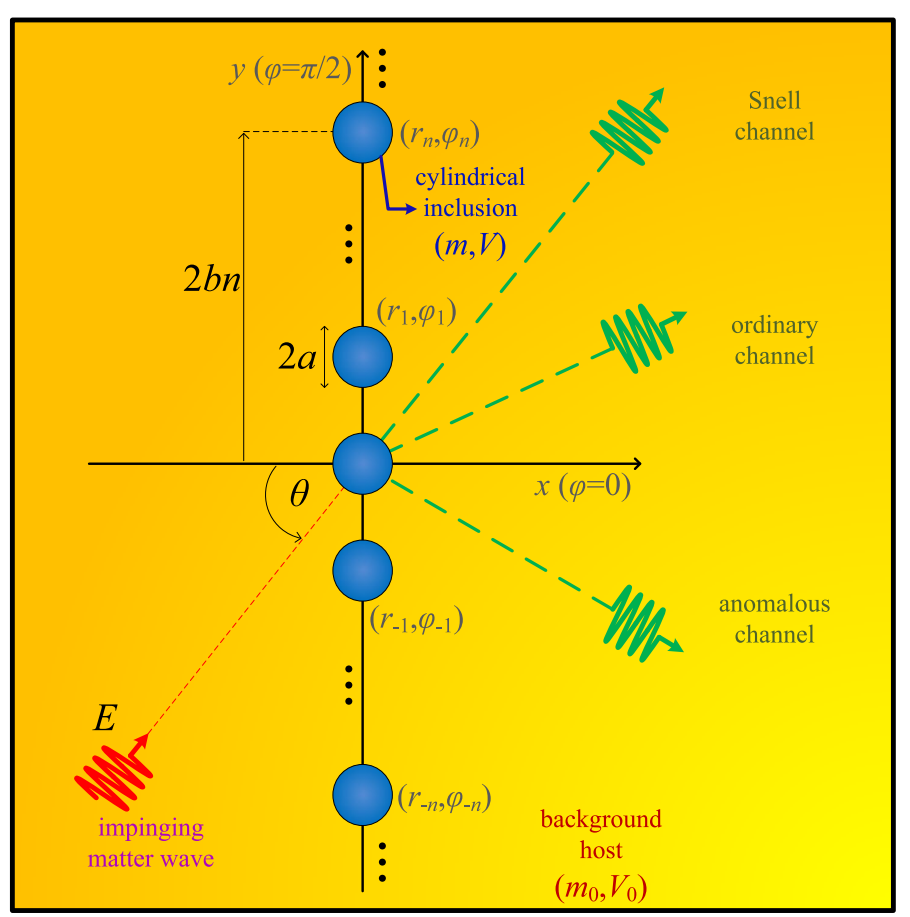

Figure 1 Setup sketch: an incident matter wave traveling into a background texture meets obliquely a periodic grating of cylindrical inclusions filled by another material. Various refractive channels are indicatively depicted

as: $\Psi_{\text {int }}^{(0)}=\sum_{p=-\infty}^{+\infty} c_{p} J_{p}(k r) e^{i p \varphi}$, where $c_{p}$ are complex determinable constants $(p \in \mathbb{Z}), J_{p}$ the Bessel function of $p$ th order and $k=\sqrt{2 m(E+\Delta V)} / \hbar$ corresponds to the wave vector norm into the cavity material. As far as the perturbation that $\Psi_{\text {back }}$ feels due to the central inclusion is concerned, it is given by: $\Psi_{\text {scat }}^{(0)}=\sum_{p=-\infty}^{+\infty} s_{p} H_{p}\left(k_{0} r\right) e^{i p \varphi}$ where $s_{p}$ are complex determinable coefficients $(p \in \mathbb{Z})$ while $H_{p}$ is the Hankel function of second type and $p$ th order.

Interestingly, due to the infinite front of the incident electron beam $\Psi_{\text {back }}$ and the infinite extent of the grating along $y$ axis, the wave function into any other $(n \neq 0)$ post and its contribution to the overall scattering effect are written via no additional coefficients than the ones of the central cylinder $\left\{c_{p}, s_{p}\right\}$. Indeed, the response of the $n$th cavity will be identical to that of the 0th one but expressed in its local coordinate system $\left(r_{n}, \varphi_{n}\right)$ and multiplied by the term $\sigma_{n}=e^{-i k_{0} 2 b n \sin \theta}$ corresponding to the phase of the incident wave at the center of $n$th inclusion; namely: $\Psi_{\text {int }}^{(n)}=\sigma_{n} \sum_{p=-\infty}^{+\infty} c_{p} J_{p}\left(k r_{n}\right) e^{i p \varphi_{n}}$ and $\Psi_{\text {scat }}^{(n)}=\sigma_{n} \sum_{p=-\infty}^{+\infty} s_{p} H_{p}\left(k_{0} r_{n}\right) e^{i p \varphi_{n}}$.

\subsection{Near field and boundary conditions}

If one employs the addition theorem for cylindrical coordinates [43, pp. 372-374], all scattering components can be expressed in terms of the central polar coordinate system $(r, \varphi)$. In particular:

$$
\Psi_{\text {scat }}(r, \varphi)=\sum_{p=-\infty}^{+\infty} e^{i p \varphi}\left\{s_{p} H_{p}\left(k_{0} r\right)+J_{p}\left(k_{0} r\right) \sum_{q=-\infty}^{+\infty} s_{q} K_{p-q}\right\},
$$


where the first term corresponds to the central inclusion $(n=0)$ and the second one to all the others. Indeed, the symbol $K_{w}$ is used for the following slowly-convergent series:

$$
K_{w}=\sum_{0 \neq n=-\infty}^{+\infty} \sigma_{n} H_{w}\left(2 k_{0} b|n|\right)(-1)^{\left(1-\frac{\operatorname{sgn}(n)}{2}\right) w} .
$$

By imposing the required boundary conditions only around the central cylinder $r=a$ for continuity of the complex-valued wave function $\Psi_{\text {int }}^{(0)}=\Psi_{\text {back }}+\Psi_{\text {scat }}$ and proportionality of its normal derivative $\hat{\mathbf{r}} \cdot \nabla \Psi_{\text {int }}^{(0)} / m=\hat{\mathbf{r}} \cdot \nabla\left[\Psi_{\text {back }}+\Psi_{\text {scat }}\right] / m_{0}$, the following linear system for the unknowns $\left\{c_{p}, s_{p}\right\}$ is formulated:

$$
\begin{aligned}
& c_{p} \frac{J_{p}(k a)}{J_{p}\left(k_{0} a\right)}-s_{p} \frac{H_{p}\left(k_{0} a\right)}{J_{p}\left(k_{0} a\right)}-\sum_{q=-\infty}^{+\infty} s_{q} K_{p-q} \\
& \quad=i^{-p} e^{-i p \theta}=c_{p} \frac{m_{0}}{m} \frac{k}{k_{0}} \frac{J_{p}^{\prime}(k a)}{J_{p}^{\prime}\left(k_{0} a\right)}-s_{p} \frac{H_{p}^{\prime}\left(k_{0} a\right)}{J_{p}^{\prime}\left(k_{0} a\right)}-\sum_{q=-\infty}^{+\infty} s_{q} K_{p-q},
\end{aligned}
$$

for $p \in \mathbb{Z}$ while the prime denotes the derivative with respect to the entire argument. The only numerical issue that may emerge in solving (3), is the evaluation of the infinite sums $K_{p-q}$ which possess oscillatory and slowly decaying general terms (as $1 / \sqrt{|n|}$ ) with respect to $n$ [44, p. 364]. To overcome such a computational snag, we exploit the analytical formula [45, p. 6]: $\sum_{n=1}^{+\infty} e^{-2 i k_{0} b(|n|+n \sin \theta)} / \sqrt{|n|}=\operatorname{Li}_{1 / 2}\left(e^{-2 i k_{0} b(1+\sin \theta)}\right)$, where $\mathrm{Li}_{1 / 2}$ is the polylogarithm function of order $1 / 2$. To put it more clearly, we subtract the asymptotic general terms of Hankel-related functions from the corresponding series (2) and we simultaneously add the aforementioned equivalent analytical result. The remainder sum converges much faster since the higher order terms of the difference vanish rapidly [46].

\subsection{Far field and diffractive channels}

Once the linear system (3) gets properly truncated with respect to $p$, the values of the angular spectral coefficients inside and outside the cylindrical inclusions $\left\{c_{p}, s_{p}\right\}$ are rigorously determined. Given the fact that the size $2 a$ of the posts is small compared to the spatial wavelength $2 \pi / k_{0}$ of the impinging wave, it is reasonable to ignore the contribution from azimuthal terms $e^{-i p \theta}$ of higher angular momentum $p$. Importantly, we are interested for the far field $(r \rightarrow+\infty)$ and the infinite dimension of the structure, combined with its symmetry, renders the effect of the scattering orders with $|p|>1$ on the final outcome, somehow less significant. More specifically, we keep the omni-directional $(p=0)$ and the bipolar $(p= \pm 1)$ variations in the scattering wave function. Let us consider the general term $W_{p}\left(k_{0} a\right)=\left|J_{p}\left(k_{0} a\right) / H_{p}\left(k_{0} a\right)\right|$ corresponding to the solution of a single impenetrable cylinder of radius $a$ embedded into a background where the impinging matter wave has wavenumber $k_{0}$. The bipolar approximation is valid as long as $W_{2}\left(k_{0} a\right) \ll \min \left\{W_{0}\left(k_{0} a\right), W_{ \pm 1}\left(k_{0} a\right)\right\}$, namely when $k_{0} a<\pi / 2$.

If one defines $s_{1 C}=s_{+1}-s_{-1}$ and $s_{1 S}=i\left(s_{+1}+s_{-1}\right)$, the quantity in (1) is written in terms of the global Cartesian coordinates $(x, y)$ for the far field $(x \rightarrow \pm \infty)$ as follows:

$$
\Psi_{\text {scat }}(x, y)=s_{0} G(x, y)-s_{1 C} \frac{\partial G / \partial x}{k_{0}}-s_{1 S} \frac{\partial G / \partial y}{k_{0}},
$$

where $G(x, y)=\sum_{n=-\infty}^{+\infty} \sigma_{n} H_{0}\left(k_{0} \sqrt{x^{2}+(y-2 b n)^{2}}\right)$. 
If one applies Poisson summation formula [47, p. 467] for the sum defining the function $G(x, y)$ and uses the Fourier pair for Hankel function indicated by [47, p. 823]: $H_{0}\left(k_{0} \sqrt{x^{2}+y^{2}}\right)=\frac{i}{\pi} \int_{-\infty}^{+\infty} \exp \left(-i \beta y-|x| \sqrt{\beta^{2}-k_{0}^{2}}\right) / \sqrt{\beta^{2}-k_{0}^{2}} d \beta$, the wave function of (4), is written as:

$$
\Psi_{\text {scat }}(x, y)=e^{-i k_{0} y \sin \theta} \sum_{u=-\infty}^{+\infty} \frac{e^{-\frac{|x|}{b} \kappa_{u}-i u \pi \frac{y}{b}}}{\kappa_{u}}\left\{i s_{0}+i s_{1 C} \frac{\kappa_{u}}{k_{0} b} \operatorname{sgn}(x)-s_{1 S}\left(\frac{u \pi}{k_{0} b}+\sin \theta\right)\right\},
$$

where $\kappa_{u}=\sqrt{\left(u \pi+k_{0} b \sin \theta\right)^{2}-\left(k_{0} b\right)^{2}}$. For $x<0$, we have reflective matter waves while for $x>0$, we obtain transmissive ones with complex amplitudes:

$$
\left\{\begin{array}{c}
R_{u} \\
T_{u}
\end{array}\right\}=\left\{\begin{array}{c}
0 \\
\delta_{u 0}
\end{array}\right\}+s_{0} \frac{i}{\kappa_{u}} \mp s_{1 C} \frac{i}{k_{0} b}-s_{1 S} \frac{\frac{u \pi}{k_{0} b}+\sin \theta}{\kappa_{u}}
$$

respectively. The first term in (6) concerns the impinging wave function in the background and $\delta_{u 0}$ is the Kronecker's delta which equals one only for $u=0$, otherwise zero.

It should be stressed that the scattering component of the wave function comprises infinite diffraction terms each characterized by an integer order $u \in \mathbb{Z}$. In Fig. 1, we schematically show three of them, every single of which corresponds to an independent refractive channel. Indeed, due to the infinite extent of the developed waves, they are linearly independent each other, namely $\int_{-\infty}^{+\infty} \int_{-\infty}^{+\infty} e^{-i\left(k_{x}-k_{x}^{\prime}\right)} e^{-i\left(k_{y}-k_{y}^{\prime}\right)} d x d y=(2 \pi)^{2} \delta\left(k_{x}-k_{x}^{\prime}\right) \delta\left(k_{y}-k_{y}^{\prime}\right)$, where $\delta$ is the Dirac's delta function and thus they can work as channels of a quantum multiport networks where information is hidden in the magnitude or phase of the wave function. The condition for the diffraction order $u$ to give a propagating mode reads:

$$
-\frac{k_{0} b}{\pi}(1+\sin \theta)<u<\frac{k_{0} b}{\pi}(1-\sin \theta),
$$

for which $\kappa_{u}$ is purely imaginary. Note that the quantity at the left-hand side of (7) is always negative and thus affects only the orders with $u<0$, while the opposite happens for the right-hand side limit (concerns diffraction of $u>0$ ). The angle at which the waves of diffraction order $u$ are traveling (either reflecting or refractive) is equal to: $\theta_{u}=\arcsin \left(\frac{u \pi}{k_{0} b}+\sin \theta\right)$. In Fig. 2(a), we represent on the plane with horizontal axis the incoming beam angle $\theta$ and with vertical axis the period of the grating divided by the traveling wavelength $k_{0} b$, the limits imposed by the inequality (7) for various positive and negative orders. These lines indicate the $k_{0} b$ (at fixed angle $\theta$ ) beyond which the diffracting channel of the related order carries a propagating wave. It is clear that responses of opposite orders $u$ are generated together (at normal incidence $\theta=0^{\circ}$ ) and as the incoming beam gets more and more oblique, it becomes easier for an anomalous wave (smaller $k_{0} b$ limit for $u<0$ ) to exist compared to an ordinary one (larger $k_{0} b$ limit for $u>0$ ). The non-blank parametric region contains $\left(\theta, k_{0} b\right)$ combinations supporting only the first anomalous order $u=-1$, except of course the inevitable Snell's response $(u=0)$. Across this "curved parallelogram", we represent the quantity $\left(\theta+\theta_{-1}\right)$ describing what is the direction spread of the anomalous reflective wave compared to the incident ray. We notice that the difference in angle can belong to an extensive value range from an anomalous direction parallel to negative $y$ axis at normal incidence $\left(\theta=0^{\circ}\right)$ to a vanishing $\theta_{u}$ at grazing ray incidence $\left(\theta=90^{\circ}\right)$. Remarkably, the two angles are opposite $\left(\theta_{-1}=-\theta\right)$ along the major diagonal 


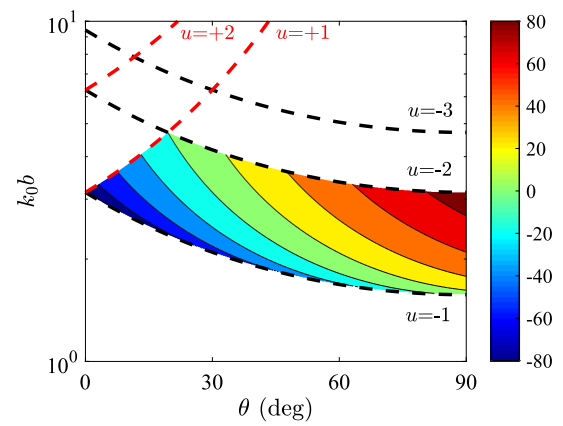

(a)

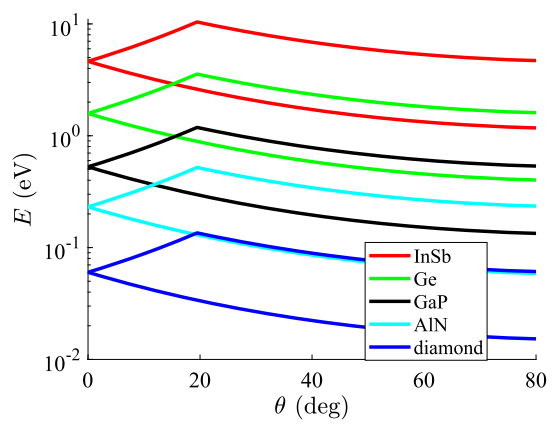

(b)

Figure 2 (a) The quantity $\left(\theta+\theta_{-1}\right)$ represented with respect to incidence angle $\theta$ and the grating period $k_{0} b$. The red and black dashed lines denote the minimum $k_{0} b$ for each $\theta$ beyond which the corresponding diffraction order gets activated. Contour plot concerns the scenario that only the orders $u=0,-1$ are propagated. (b) The upper and lower limits for the energy $E$ of the impinging particle for which only the first anomalous order $u=-1$ (plus the Snell's one, $u=0$ ) is guided according to (7), as function of incidence angle $\theta$ for various host media $(b=2.5 \mathrm{~nm})$

of the colored domain defined by the limits of orders $u= \pm 1$ and $u=-2$; such a feature means that the electron beam is reflected back to the source that created it $(x<0)$ and negatively refracted [48] from the other side $(x>0)$ of the grating.

When it comes to the probabilities for the particle to travel along the reflective or transmissive direction defined via angle $\theta_{u}$, they are given respectively by [49, p. 38]:

$$
\rho_{u}=\left|R_{u}\right|^{2} \frac{\cos \theta_{u}}{\cos \theta}, \quad \tau_{u}=\left|T_{u}\right|^{2} \frac{\cos \theta_{u}}{\cos \theta} .
$$

Indeed, the normalization condition stands only along the direction being normal $(x)$ to the cluster of cylindrical cavities.

\section{Numerical results}

\subsection{Optimization strategy}

By inspection of (7), it is clear that the wave with $u=0$ is always propagating since it is dictated by the Snell's law [42, pp. 187-191] formed for electromagnetic fields. However, the response is quite trivial since it partially expresses the unhampered propagation of the incoming e-beam in the absence of the grating and partially the reflection achieved with a perfect mirror. On the contrary, when $u \neq 0$, namely when the distance $k_{0} b$ or the angle $\theta$ increases, we obtain modes steered at unconventional, counter-intuitive directions that can be directly exploitable to quantum circuits and quantum signal processors. If one keeps $\theta>0$ without losing the generality since the layout is symmetric with respect to $x$ axis, it is understood that the first diffraction wave that can be activated beyond ordinary Snell's response (as $k_{0} b$ or $\theta$ increases) is the one with order $u=-1$; usually this correspond to negative diffraction direction $\left(\theta_{-1}<0\right)$ and thus is called anomalous. Three characteristic cases for the transmissive channels are indicated in Fig. 1, where the Snell $\left(\theta_{0}=\theta\right)$, an ordinary $\left(\theta_{u}>0\right)$ and an anomalous $\left(\theta_{u}<0\right)$ refraction are sketched.

To keep the objective of this study as simple as possible, we will confine ourselves to designs supporting only the ordinary Snell-like response $(u=0)$ and the anomalous order $u=-1$. In particular, we aim at finding gratings that suppress the conventional reflection 
and transmission $\left(\rho_{0}, \tau_{0}\right)$ and inevitably boost the matter wave steering along the anomalous direction $\left(\rho_{-1}, \tau_{-1}\right)$. Note that the condition for zero Snell's response reads:

$$
\rho_{0}=\tau_{0}=0 \quad \Rightarrow \quad s_{0}=2 s_{+1} \sin \theta=2 s_{-1} \sin \theta
$$

Our optimization scheme takes a long directory of $M$ quantum media (with macroscopic potentials and effective masses) containing from isolated elements (germanium, silicon, carbon etc.) and semiconductors (arsenides, nitrides, tellurides etc.) to arbitrary alloys, mixtures and heterojunctions [50, pp. 229-245]. Sophisticated computational simulation models evaluating the effective parameters [51, pp. 75-80] that describe the transport of quantum particles into them have been developed [52], being additionally validated by high-fidelity measurements. It should be stressed that the effective masses of the materials are positive which means that the waves into them are either of purely propagating or purely evanescent nature. Note that masses can be different when the particle moves along different directions, namely the material is considered anisotropic, a property commony assumed for similar setups in Electrodynamics [53]. Every single of the $M^{2}>1000$ combinations is tried by assigning to each material from the list, both the role of background host and the filling to the cylindrical cavities. Every single of the $M^{2}$ designs is examined by carefully sweeping the angle of the incoming matter wave over an extensive range excluding very oblique beams: $5^{\circ}<\theta<85^{\circ}$, the ratio between the inclusion radius over the half-period of the grating: $0.1<a / b<0.4$ and the kinetic energy $E$ of the incident particles by respecting (7) for single anomalous propagating diffraction order $u=-1$. If one keeps the half-distance between the inclusions $b$ fixed, the permissible value range for $E$ at a specific $\theta$ is given by the pair of lines of the same color in Fig. 2(b), each of which corresponds to a different background medium with a different effective mass $m_{0}$. Such an interval of energies giving only anomalous diffraction of $u=-1$ (plus the Snell's $u=0$ ), shrinks with the effective mass $m_{0}$ (in the case of diamond, $m_{0}$ is the highest) even though it is not apparent due to the logarithmic scale of energy axis. Note that, exactly as happens in Fig. 2(a), the vertical extent of the acceptable parametric domain initially increases with $\theta$ up to the breaking point that the generating loci of the two diffraction orders $(u=-2$ and $u=+1)$ cross each other, namely at $\theta=\arcsin (1 / 3) \cong 19.5^{\circ}$. Beyond this point, the considered $E$ range becomes smaller with $\theta$.

By using a tight brute-force optimization scheme, our aim is to determine the cylinder size $a / b$ (for constant $b$ ) that pairs better with the impinging beam of $\{\theta, E\}$ leading to maximal anomalous reflection $\rho_{-1}$ or transmission $\tau_{-1}$ from (8), by approximately obeying the condition (9).

\subsection{Optimal designs response}

From all the parameters defining the configuration of Fig. 1, once the pair of materials has been decided, the one changing less easily is the half-period of the grating $b$, since even the size $a$ of the cavities is controllable during fabrication process, let alone the incidence angle $\theta$ and the impinging particles energy $E$. Indeed, regardless of the construction philosophy (bottom-up [29] or top-down [30]) or the assembly nature (physical [29] or chemical [27]), the grid at which the inclusions are planted is predetermined. Therefore, when we implementing our optimization strategy, we assume a fixed $b$ throughout $(2 b=5 \mathrm{~nm}$ in Figs. 3(a) and 3(b), $2 b=10 \mathrm{~nm}$ in Figs. 3(c) and 3(d)), without varying it simultaneously. More specifically, the results of the proposed inverse process are shown in Fig. 3, 


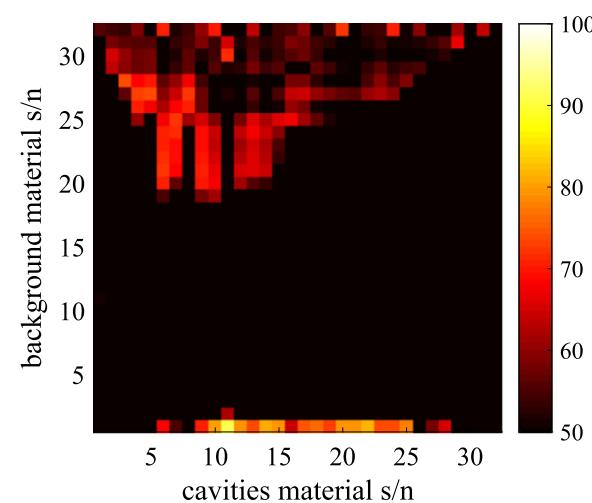

(a)

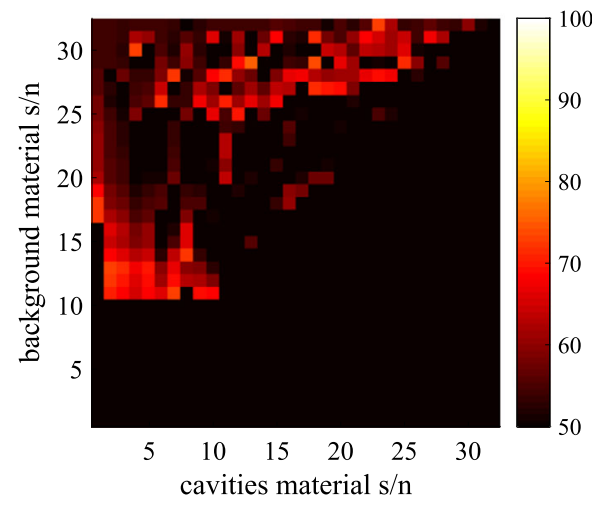

(c)

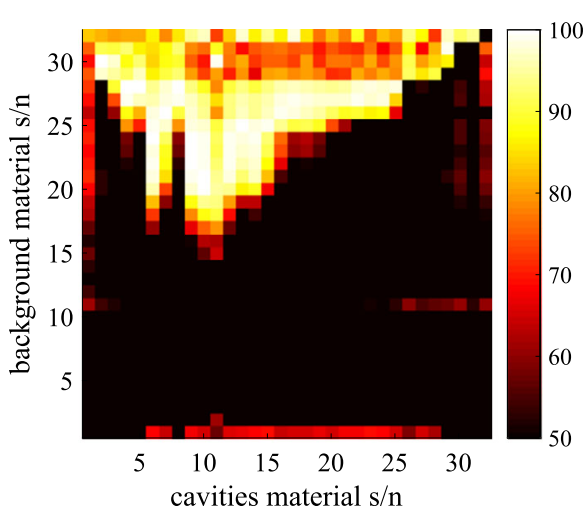

(b)

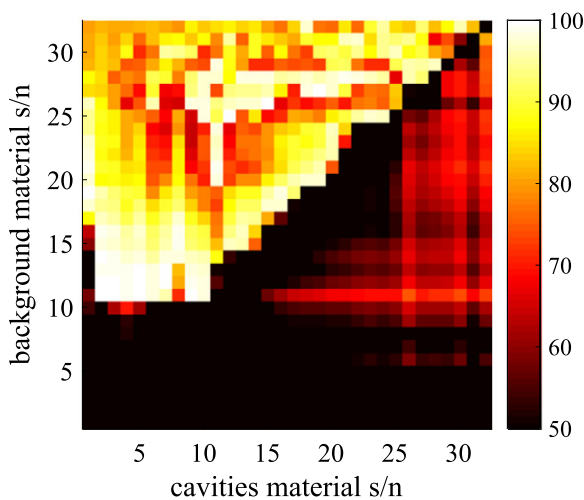

(d)

Figure 3 Percentage of anomalous diffraction of optimal designs for various cavities materials (horizontal axis) and various background hosts (vertical axis). The media have been sorted with increasing potential energy: larger potential, larger serial number $(s / n)$. Designs supporting only two diffracted orders $(u=0,-1)$ maximizing $(\mathbf{a}),(\mathbf{c})$ anomalous reflection probability $\rho_{-1},(\mathbf{b}),(\mathbf{d})$ anomalous transmission probability $\tau_{-1}$, with: (a), (b) $b=2.5 \mathrm{~nm}$ and (c), (d) $b=5 \mathrm{~nm}$

where to each quantum material from our list (with $M=32$ entries) has been assigned a serial number $(\mathrm{s} / \mathrm{n})$ in proportion to how big is its macroscopic potential energy $\left(V\right.$ or $\left.V_{0}\right)$. Along the horizontal axis, we show the serial number of the material filling the cylindrical nanocavities, while the vertical axis determines the texture of the background host. In this way, a square canvas of $M^{2}=32^{2}=1024$ pixel is formed the lower right triangular part of which corresponds to quantum barrier configurations while the upper left one describes quantum well setups.

In Fig. 3(a), the represented quantity is the maximal anomalous reflection probability $\rho_{-1}$ when the rest of parameters $\{a / b, \theta, E\}$ are swept as indicated above; poor results $\left(\rho_{-1}<50 \%\right)$ are omitted. We clearly observe that most of the successful designs appear at the upper left region of our materials plane, namely concern quantum well configurations giving quite high anomalous steering of the reflective wave $\left(\rho_{-1}>70 \%\right)$. However, the best score is achieved for quantum barriers configurations, namely when the background is the material of the lowest potential $V$ (InSb); for certain inclusion media surpasses the limit of 90\%. In Fig. 3(b), we show the results of the optimization when maximizing the transmissive probability $\tau_{-1}$; one directly observes much higher performances compared to the case of $\rho_{-1}$ in Fig. 3(a). Again the quantum well designs are much more efficient 
than the corresponding quantum barrier ones and in most examples the maximal performance reach the perfect outcome of $100 \%$. Overall there are more combinations of materials that give significant anomalous transmission $\left(\tau_{-1}>50 \%\right)$ compared to them excelling at anomalous reflection $\left(\rho_{-1}>50 \%\right)$; however, the configurations hosted in InSb exhibit much less granted response. In Figs. 3(c) and 3(c) we consider a larger period of the cylinders grating $(2 b=10 \mathrm{~nm})$ and repeat the maximization process described above. As far as the reflective probability is concerned, the average maximal score is lower than those in Fig. 3(a); nonetheless, there are more alternative texture pairs that deliver above the $50 \%$ threshold, most of which with low $V_{0}$. Such a trend for more degrees of freedom when $b$ is larger, has been verified by Fig. 3(d), where the maximal $\tau_{-1}$ is shown; however, the performance remains as high as in Fig. 3(b) this time. Importantly, multiple material combinations corresponding to quantum barrier setups emerge and give acceptable anomalous transmission, while the best results are recorded for those hosts of relatively diminished potential energy.

In Fig. 4, we show the response along the anomalous direction of certain designs from Figs. 3(a), 3(b) for various incidence angles $\theta$ and variable impinging energies $E$; results are shown for those $(\theta, E)$ combinations that only $u=0,-1$ diffraction orders are excited. In Fig. 4(a), we consider the anomalously reflecting design of InP posts embedded in BeTe

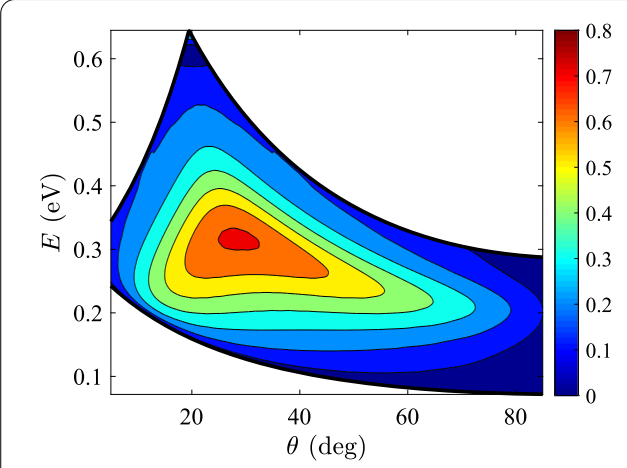

(a)

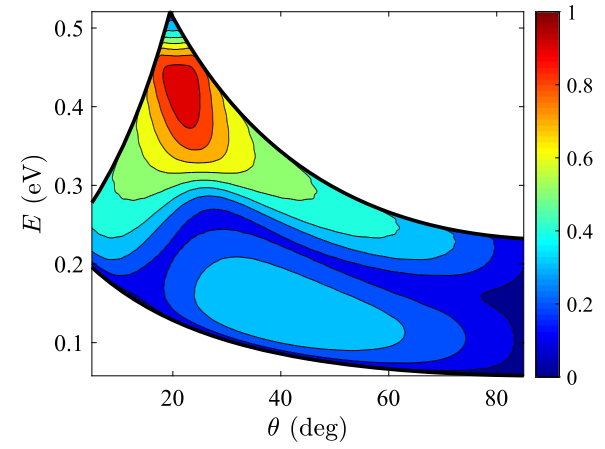

(c)

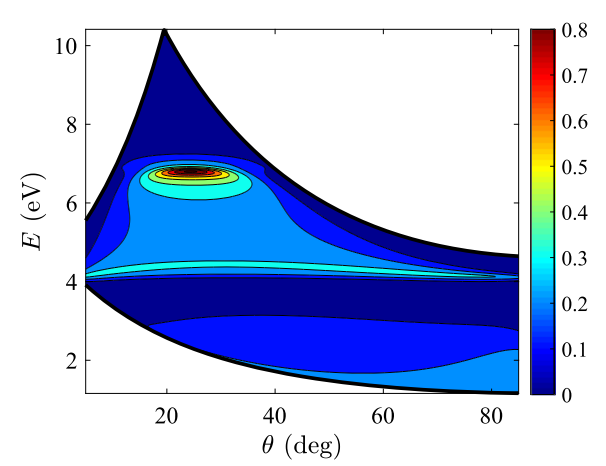

(b)

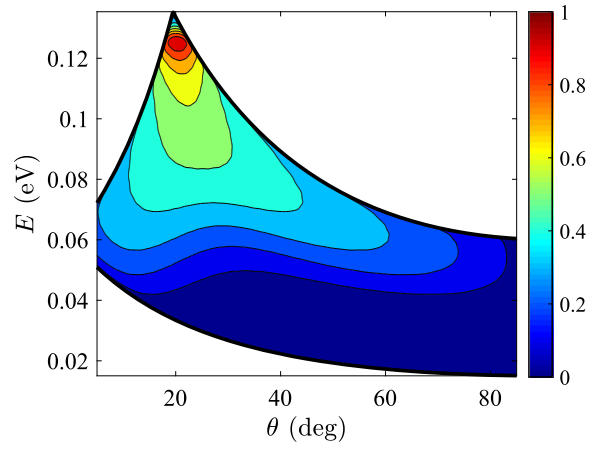

(d)

Figure 4 Anomalous diffraction probability of selected optimal designs of Figs. $3(a)$ and $3(b)(b=2.5 \mathrm{~nm})$ as function of the angle of impinging electrons $\theta$ and their kinetic energy $E$. Only results at combinations $(\theta, E)$ leading to exactly one anomalous diffraction order $(u=-1)$ are shown. (a) $\rho_{-1}$ for optimal InP inclusions in BeTe background (Fig. 3(a), quantum well, $a / b=0.40, k_{0} a \cong 1.32$ ), (b) $\rho_{-1}$ for optimal CdO inclusions in InSb background (Fig. 3(a), quantum barrier, $a / b=0.36, k_{0} a \cong 1.39$ ), (c) $\tau_{-1}$ for optimal CdTe inclusions in AIN background (Fig. 3(b), quantum well, $\left.a / b=0.28, k_{0} a \cong 1.19\right)$, (d) $\tau_{-1}$ for optimal SiC inclusions in diamond background (Fig. $3\left(\right.$ b) , quantum well, $a / b=0.36, k_{0} a \cong 1.63$ ) 
(from Fig. 3(a)). The maximization of the represented quantity $\rho_{-1}$ occurs in the middle of the map, while it vanishes close to the borders generating diffraction of $u=+1$ or $u=-2$; however, when one crosses parametrically such a boundary may experience huge increase in high-order response. In Fig. 4(b), we regard the highest-performing quantum grating, namely CdO cylinders into InSb (from Fig. 3(a)) with $\rho_{-1}>90 \%$. The operational range of energies $E$ is much larger since the effective mass of InSb host $m_{0}$ is tiny (see Fig. 2(b)); in addition, the resonance is substantially sharper than the one of Fig. 4(a) even though it occurs in a similar $E$-interval.

In Fig. 4(c), we move to maximally- $\tau_{-1}$ setups and examine the optimal CdTe cavities in AlN background (from Fig. 3(b)). We notice that the peak is exhibited for $\theta \cong \arcsin (1 / 3)$ and close to the maximal permissible energy. Furthermore, the represented quantity does not vanish when higher diffractive orders are developed while a secondary local maximum appears at lower $E$ for $\theta \cong 45^{\circ}$. Finally, in Fig. 4(d), we investigate the response of $\mathrm{SiC}$ rods into diamond (from Fig. 3(b)). The resonance emerges at even more significant energies, close to the point that the diffraction orders $u=-2,+1$ are simultaneously activated and $\tau_{-1}$ drops more rapidly far from it.

In Fig. 5, we investigate the influence of incident energy $E$ sweep on all the reflective and transmissive probabilities for certain designs. Firstly, we consider the layout of Fig. 4(b) supporting substantial $\rho_{-1}$ and, indeed, the corresponding term gets sharply maximized at $E \cong 6.5 \mathrm{eV}$. In Fig. 5(a), where the $\rho_{u}=\rho_{u}(E)$ curves are represented, one can identify the energy thresholds at which each diffraction order $u$ gets activated. Moreover, the rapid

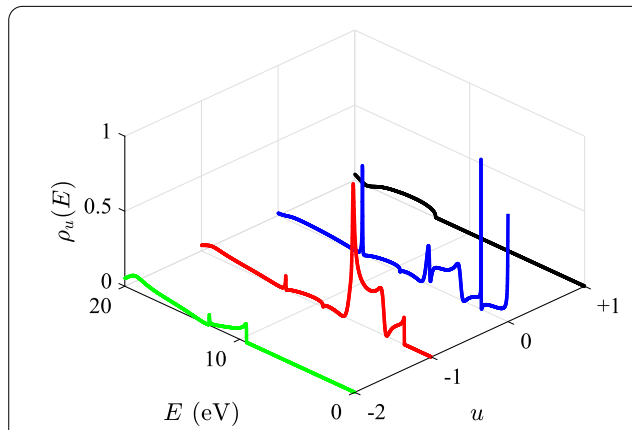

(a)

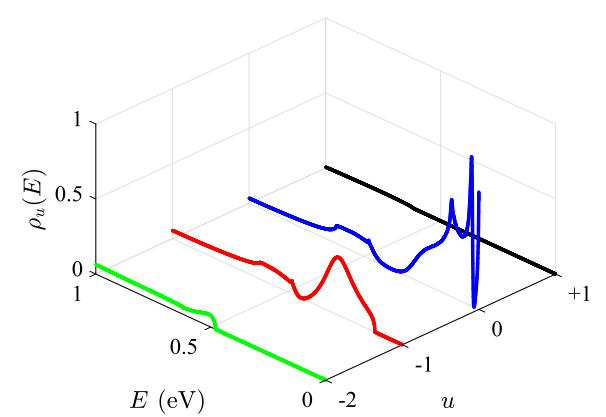

(c)

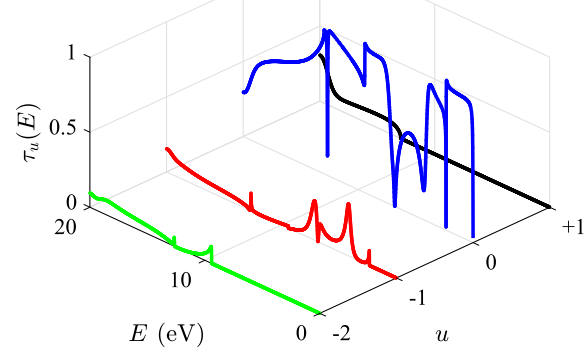

(b)

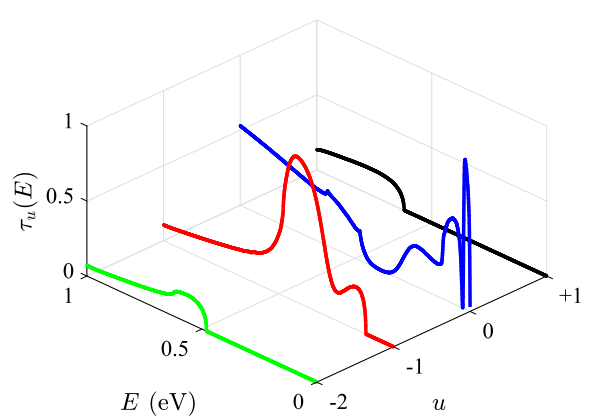

(d)

Figure 5 Reflective $\rho_{u}$ and transmissive $\tau_{u}$ probabilities for various diffraction orders $u$ as functions of energy level $E$ for: (a), (b) optimal CdO inclusions in InSb background (Fig. 4(b), maximal $\rho_{-1}$ ) and (c), (d) optimal CdTe inclusions in AIN background (Fig. 4(c), maximal $\left.\tau_{-1}\right)$. Incidence angles $\theta$ are selected so that maximal anomalous diffraction is achieved 
variation of $\rho_{0}$ is complementary to that of $\tau_{0}$ in Fig. 5(b) at low- $E$ range, since the configuration is lossless and the matter waves are not channeled towards alternative (except for Snell's) directions. By inspection of Figs. 5(a) and 5(b), one can understand that the proposed structure can work as a multichannel functional quantum metasurface being able to control incoming and outgoing matter waves in a number of propagation directions simultaneously. In this way, the concept of engineering multichannel reflectors [24] that can control incoming and outgoing waves in a number of propagation directions simultaneously, which has been elaborated and established in Photonics, is translated into quantum arena. In Figs. 5(c) and 5(d), we regard a design with substantial $\tau_{-1}$ and, once again, the activation of more diffractive orders $u$ for increasing $E$, is noticed. Furthermore, highly selective responses are observed for $\left\{\rho_{0}, \tau_{0}\right\}$ and at energy levels that new orders are generated, abrupt changes are exhibited. Finally, the maximum in $\tau_{-1}$ is more wide-band compared to the corresponding one in $\rho_{-1}$ of Fig. 4(a), which is also understood by comparing Fig. 3(b) with 3(c).

In Fig. 6(a), we examine an optimal layout from the ones reported in Fig. 3(c) (ZnTe cavities hosted by $\mathrm{SiC})$, where the period of the grating is larger $(b=5 \mathrm{~nm})$. Similarly to Figs. 4(a) and 4(b), the maximum occurs in the middle of the curved rectangle and diminishes rapidly when one moves towards the point of activation of the two higher-order diffraction waves $(u=+1,-2)$ at $\theta=\arcsin (1 / 3)$. The huge decline pace for increasing energy $E$ is potentially related to the fact that optimization gives an $a / b=0.1$ corresponding to an extremum of the considered interval $(0.1<a / b<0.4)$; the same happens in Fig. 4(b) where $a / b=0.4$. In order to avoid reporting resonances appearing outside of the regarded

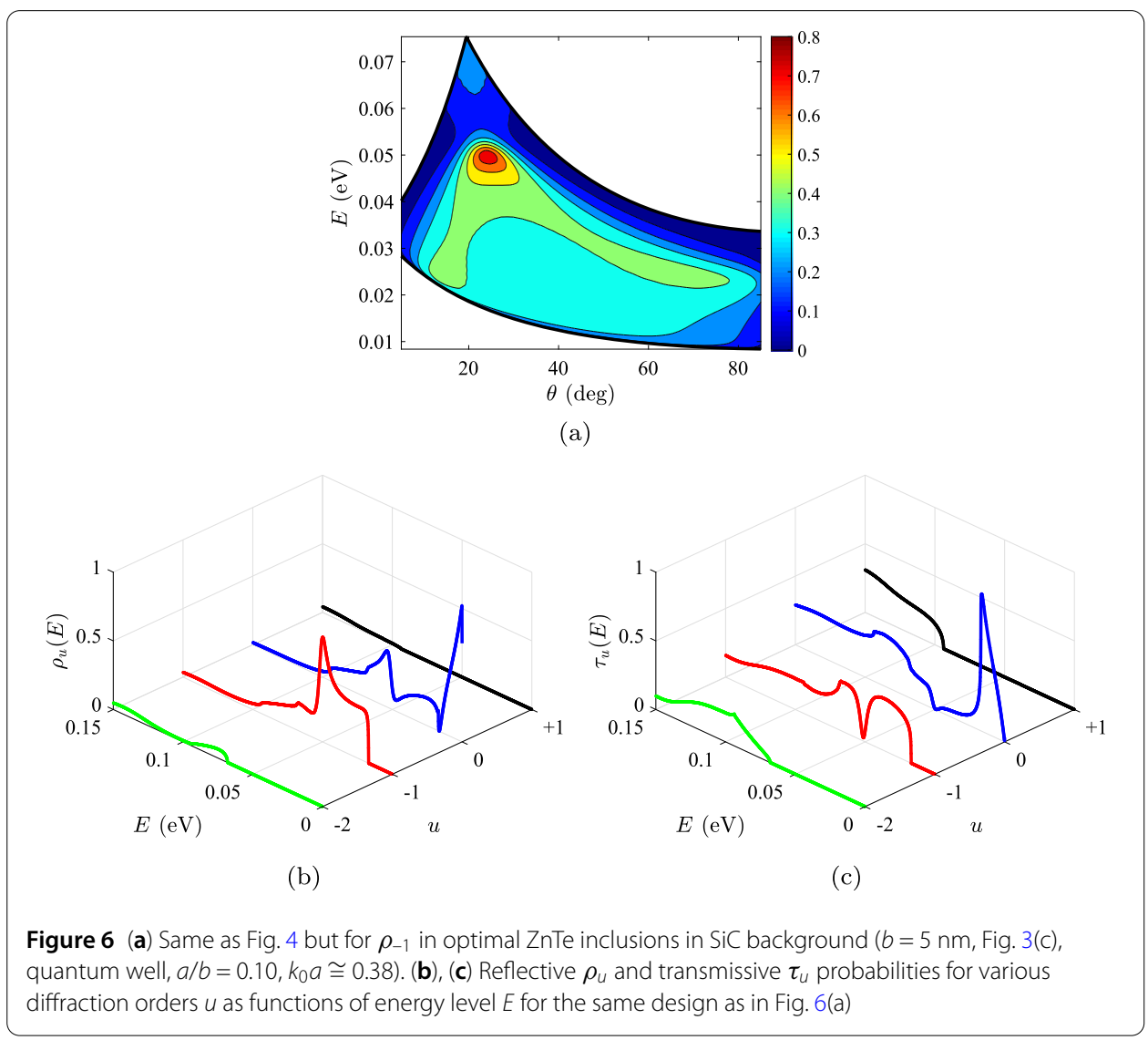


parametric box, a refinement to the adopted optimization strategy should be performed as in similar photonic studies [54]. In Fig. 6(b), we observe the variations of reflective probabilities $\rho_{u}$ for various orders $u$ as functions of incoming particles energy $E$ for the same design, while in Fig. 6(c), the corresponding transmissive probabilities $\tau_{u}=\tau_{u}(E)$ are shown. The utility of the proposed configuration as a multi-port quantum network that steers the electron beams with controllable direction and intensity, is once again demonstrated; note also the large values recorded for the transmissive probability $\tau_{u}$ for the orders with $u=+1,-2$.

\subsection{Performance robustness}

As briefly mentioned above, there are several alternative fabrication methods for the setup depicted in Fig. 1, namely growing multiple parallel nanowires into a specific host. Chemical approaches constitute a major construction category containing vapor-liquid-solid growth after injecting precursor gases leading to the nucleated development of cavities from catalytic particles [55] and fast modulation of nanowire composition during bottomup engraving combined with wet-chemical etching [27]. In addition, a highly-efficient solution-based method for the direct growth of core/shell nanowire arrays on various conductive substrates is implemented [56] suitable for various applications in electrochemical energy storage and optical devices. Epitaxial techniques are also utilized for physical creation of core-multishell structures of radial heterostructure growth for the development of nanowire-based structures [28] or bottom-up syntheses of complex quantum devices with a special focus on nanowire networks with a predefined number of superconducting islands [29]. Furthermore, top-down practices have been applied for realizing diamond cavities in bulk hosts with use of inductively coupled plasma etching [30], while semiconductor processing techniques are implemented to give photonic crystal heterostructures and interfaces, namely the quantum analogue of metasurfaces [57]. Finally, self-assembling design rules are being developed for the generation of complex hierarchical nanostructured systems by exploiting the analogy of nanoparticles with molecules leading to robust and highly reproducible synthesis [58], while micromachined cavities are successfully employed for the construction of superconducting resonators in various quantum layouts [59].

However, all the aforementioned approaches are not flawless and thus the obtained designs contain several size imperfections; either the radius $a$ of the rods is not exactly equal to the optimal one or (less frequently) the recommended half-period $b$ is not properly respected. In both cases, the duty cycle $a / b$ is different from that indicated by the optimization process; therefore, it is meaningful to examine the response of the device when the ratio $a / b$ is perturbed around the proposed value. In Fig. 7, we consider maximally reflecting designs from Fig. 3(a) and show the trajectory on the reflection $\left(\rho_{0}, \rho_{-1}\right)$ and transmission $\left(\tau_{0}, \tau_{-1}\right)$ plane where the horizontal axis corresponds to the Snell's diffraction and the vertical the anomalous quantity of order $u=-1$. If further diffraction waves are propagated, they are evaluated by subtracting the values $\left\{\rho_{0}, \rho_{-1}, \tau_{0}, \tau_{-1}\right\}$ from unity indicated by the dashed diagonal lines. In Fig. 7(a), where the reflective channels are examined, one notices the remarkable robustness of the design with InP cavities into BeTe and the sharp change in the performance of BeTe cylinders into diamond; similar trends are recorded in Fig. 7(b), where the transmissivities are represented. As far as the most successful design is concerned ( $\mathrm{CdO}$ rods in $\mathrm{InSb}$ ), we observe a remarkable transition 


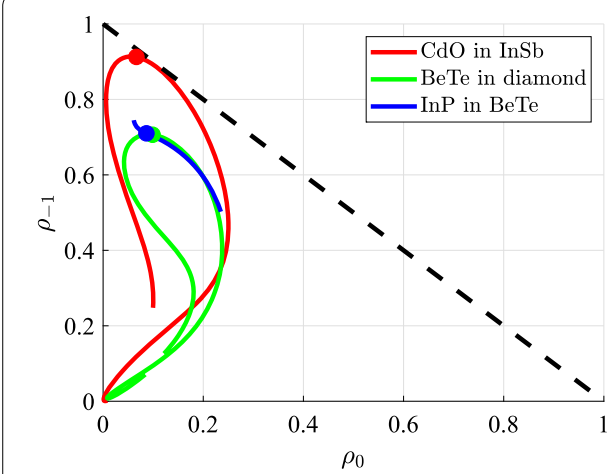

(a)

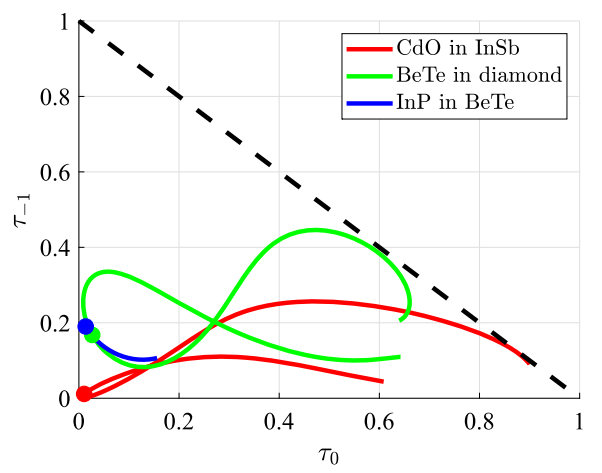

(b)

Figure 7 Trajectories of various designs (with $a=2.5 \mathrm{~nm}$, Fig. 3(a), support maximal anomalous reflection) response on the plane of Snell's (horizontal) and first anomalous (vertical) diffraction as the duty cycle $a / b$ perturbs by $\pm 10 \%$ around the optimal value. (a) Loci on $\left(\rho_{0}, \rho_{-1}\right)$ plane and $(\mathbf{b})$ Loci on $\left(\tau_{0}, \tau_{-1}\right)$ plane. Markers denote the optimal operation point and dashed lines indicate the maximum quantity, equaling to one

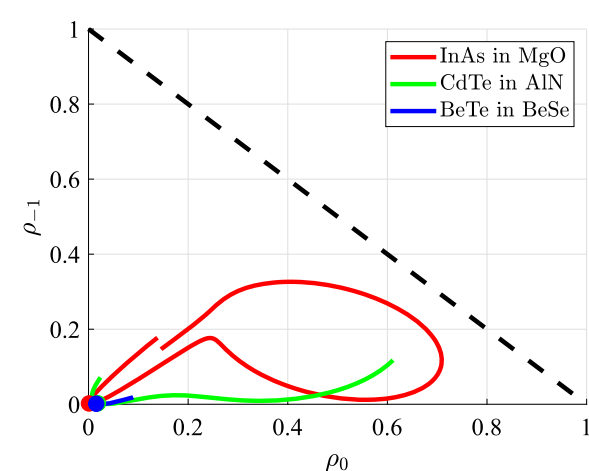

(a)

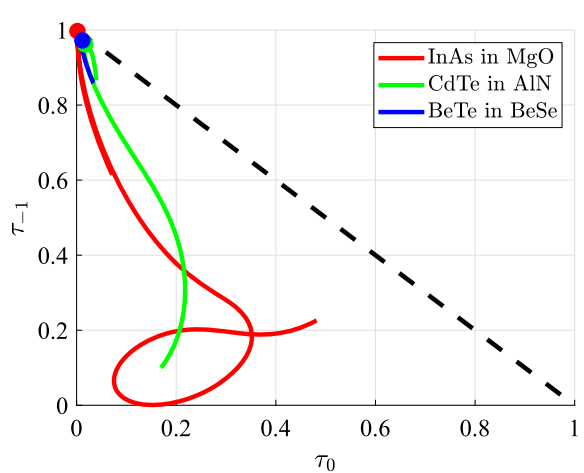

(b)

Figure 8 Same as in Fig. 7 for designs appearing in Fig. 3(b) that support maximal anomalous transmission

from a fully reflective regime (optimal operational point of Fig. 7(a)) to a fully matched one at the point that the red curve touches the dashed line in Fig. 7(b).

In Fig. 8, we show the same quantities as in Fig. 7 but for three maximally transmissive designs appearing in Fig. 3(b). The optimal operational regimes are almost fully transmissive and that is why the markers are located around the origin in Fig. 8(a) and around the upper left corner in Fig. 8(b). Similarly to Fig. 7, we notice the different robustness of the three picked designs and the asymmetry of the curves around the maximal anomalous transmission points; indeed, the response of the structure changes much more reluctantly when $a / b$ is selected bigger than the recommended compared to the opposite way. It must be finally stressed that every single point on the curves corresponds to a distinct set of probability separations along specific directions of space and thus defines a distinct point of operation of the formed quantum multiport network.

Therefore, we are permitted to say that even when robustness of the design is not substantial, its utility gets not necessarily reduced: as long as the redistribution of probabilities is rigorously computed, any combination of parameters can lead to a useful response profile. In other words, not only the ratio $a / b$ but even if the angle of incidence $\theta$ or the particle 


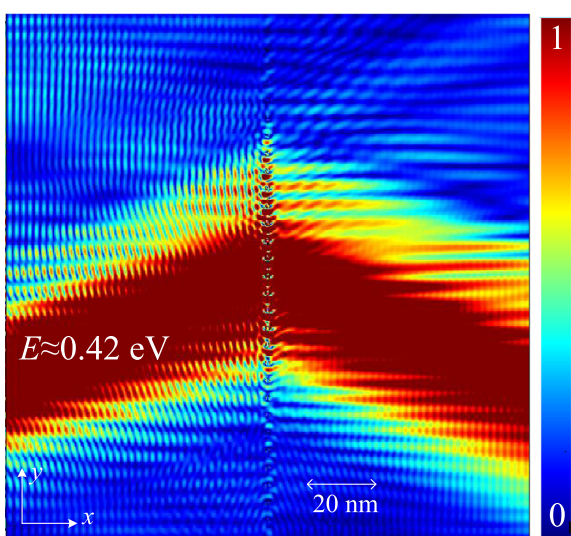

(a)

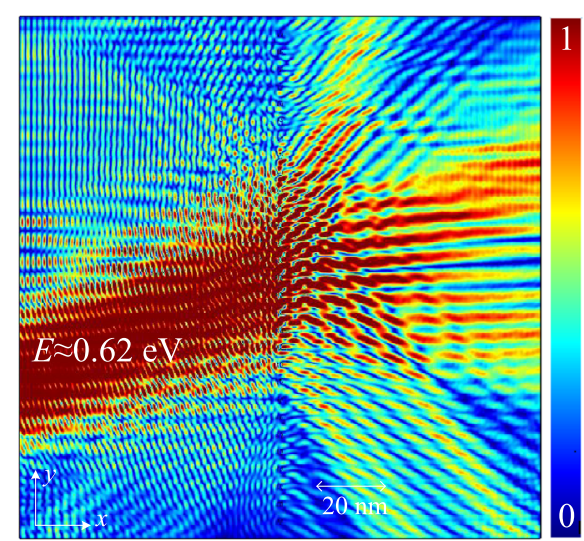

(b)

Figure 9 Spatial distribution of normalized $|\Psi(x, y)|^{2}$ for the design of Fig. 4(c) with (a) $E \cong 0.42 \mathrm{eV}$ (optimal anomalous transmission) and (b) $E \cong 0.62 \mathrm{eV}$. Same scale is used for both figures

energy $E$ is misselected, can give modules with interesting channel outline serving alternative needs into a quantum circuit. In Fig. 9, we consider the design of Fig. 4(c), namely CdTe cavities in AlN background, and show the spatial distribution of $|\Psi(x, y)|^{2}$, with help from commercial software. In Fig. 9(a), the energy of the incoming particle is exactly equal to that achieving maximal anomalous refraction and such an effect is fully demonstrated by the abrupt turn of the propagation direction towards the same side that the metasurface is illuminated. In Fig. 9(b), we have selected a different energy for the incoming particle and we obtain multiple (4-5) refractive matter waves with different angles, waist widths and intensities; in this way, the operation of the quantum grating as multiport channel network with rich scattering matrix is shown. These "fringes", namely the slightly "noisy" pattern of Fig. 9, are attributed to the fact that both the front of the incoming beam and the metasurface extent are finite.

A case of special interest in the normal incidence $\left(\theta=0^{\circ}\right)$, which demands a specific level of energy $E \gtrsim \frac{1}{2 m_{0}}(\hbar \pi / b)^{2}$ for anomalous diffraction (this time both $u= \pm 1$ orders are activated). In Fig. 10(a), we show the probability distribution $|\Psi(x, y)|^{2}$ for a normally excited design which achieves an almost perfect splitting of the incoming beam along two symmetric directions corresponding to the two simultaneously (for $\theta=0^{\circ}$ ) generated diffraction orders with $u= \pm 1$. In this way, the functionality of the regarded layout as wave matter splitter is fully demonstrated. Note that the intensity of the refractive beams is controllable via the texture and the rest of the parameters of the configuration. In Fig. 10(b), we show a scenario of splitting with reduced magnitude controlled by the mechanism of normal reflections $\rho_{0}$.

\section{Conclusions}

Diffraction of matter waves along various counter-intuitive directions with controllable intensity is an important and general process behind numerous quantum systems. Perhaps the simplest structure that such an anomalous effect may occur is a periodic metasurface comprising cylindrical posts embedded into a background host; thus, in this study its optimal working regimes for various combinations of quantum media are reported. The operation of the structure as a quantum multiport network, where each diffraction order 


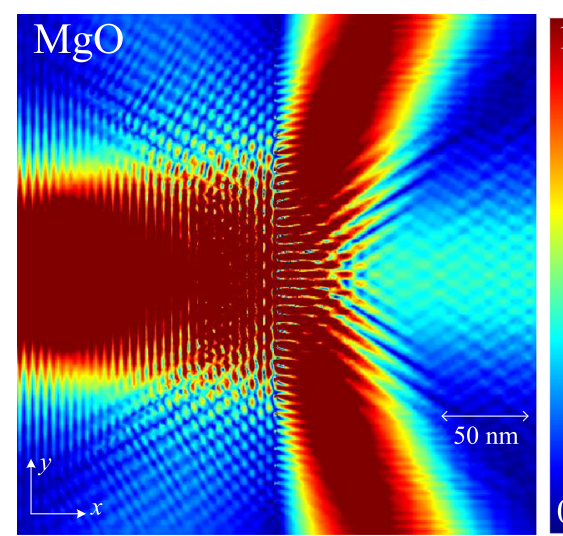

(a)

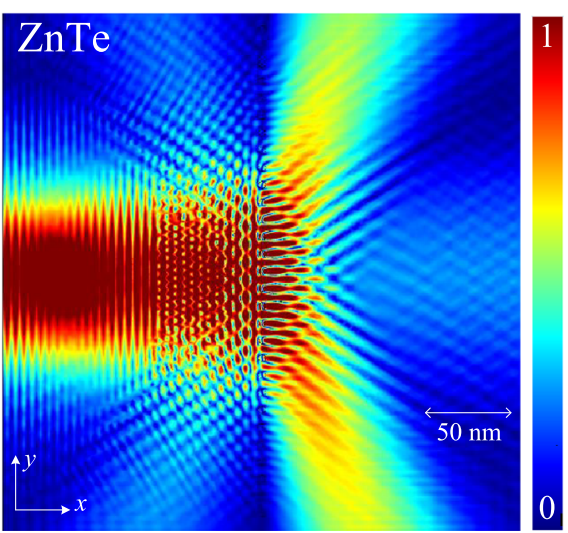

(b)

Figure 10 Spatial distribution of normalized $|\Psi(x, y)|^{2}$ at normal incidence $\left(\theta=0^{\circ}\right)$ for: (a) BP rods in MgO: perfect beam splitting, (b) BAs rods in ZnTe: controllable partial beam splitting

plays the role of a channel of information, is demonstrated at an extensive range of incoming e-beam angles and energies while the effect of the inclusions size on the re-distribution of probabilities is examined.

The presented results are also useful to the interested experimentalist that aims at fabricating steering modules for quantum circuits and signal processors, where the spatial channeling of matter waves is required. In this way, multiple alternative materializations for a fundamental and generic subsystem vital for several quantum engineering applications are provided, ready to respect the availability and feasibility constraints. Interesting expansions of the present work may contain similar concepts already implemented in Electromagnetics like inhomogeneous cylinders [60], rods placed into a different host [61] or wires of unknown texture that can be guessed [62] via external measurements.

\section{Acknowledgements}

The author is grateful to Prof. Efthimios Kaxiras (Harvard University, Cambridge, MA, USA) for useful discussions regarding the quantum interactions between matter and electron beam.

\section{Funding}

This work was partially supported by Nazarbayev University Faculty Development Competitive Research Grant 021220FD4051 entitled: "Optimal Design of Photonic and Quantum Metamaterials".

\section{Availability of data and materials}

The datasets used and analyzed during the current study are available from the corresponding author upon reasonable request.

\section{Competing interests}

The author declares that they have no competing interests.

\section{Authors' contributions}

CV as the sole author of the manuscript, conceived, designed and performed the analysis; he also wrote and reviewed the paper. The author read and approved the final manuscript.

\section{Publisher's Note}

Springer Nature remains neutral with regard to jurisdictional claims in published maps and institutional affiliations.

Received: 13 October 2020 Accepted: 28 January 2021 Published online: 06 February 2021

\section{References}

1. Pirandola S, Bardhan BR, Gehring T, Weedbrook C, Lloyd S. Advances in photonic quantum sensing. Nat Photonics. 2018;12:724 
2. Ajoy A, Liu Y-X, Saha K, Marseglia L, Jaskula J-C, Bissbort U, Cappellaro P. Quantum interpolation for high-resolution sensing. Proc Natl Acad Sci USA. 2017;114:2149.

3. Zaiser S, Rendler T, Jakobi I, Wolf T, Lee S-Y, Wagner S, Bergholm V, Schulte-Herbruggen T, Neumann P, Wrachtrup J. Enhancing quantum sensing sensitivity by a quantum memory. Nat Commun. 2016;7:12279.

4. Clausen C, Usmani I, Bussières F, Sangouard N, Afzelius M, de Riedmatten H, Gisin N. Quantum storage of photonic entanglement in a crystal. Nature. 2011:469:508.

5. Arute F et al. Quantum supremacy using a programmable superconducting processor. Nature. 2019;574:505.

6. Zhong H-S et al. Quantum computational advantage using photons. Science. 2020;370:1460.

7. Kimble HJ. The quantum Internet. Nature. 2008;453:1023.

8. National strategic overview for quantum information science. Product of the Subcommittee on Quantum Information Science under the Committee on Science of the National Science and Technology Council. 2018. https://www.gao.gov/assets/700/694749.pdf.

9. Acín A et al. The quantum technologies roadmap: a European community view. New J Phys. 2018;20:080201.

10. Lloyd S, Englund D. Future directions of quantum information processing. In: Proceedings of workshop on the emerging science and technology of quantum computation, communication, and measurement. 2018. https://basicresearch.defense.gov/Portals/61/Documents/future-directions/Future_Directions_Quantum.pdf?ver= 2017-09-20-003031-450

11. Considerations for maintaining U.S. competitiveness in quantum computing, synthetic biology, and other potentially transformational research areas. Report to the Subcommittee on Research and Technology, Committee on Science, Space, and Technology, USA House of Representatives, GAO-18-656. 2018. https://www.gao.gov/assets/ 700/694749.pdf.

12. Raymer MG, Monroe C. The US national quantum initiative. Quantum Sci Technol. 2019:4:020504

13. Ab-initio solid-state quantum materials: design, production, and characterization at the atomic scale. Multi-university research initiative 2018-2023, PI: Dirk Englund, Massachussets Institute of Technology. 2018.

14. Scalable certification of quantum computing devices and networks. Multi-university research initiative 2017-2022, PI: Thomas Vidick, California Institute of Technology. 2017.

15. Quantum state control of molecular collision dynamics. Multi-university research Initiative 2019-2024, PI: Arthur Suits, University of Missouri. 2019

16. Politi A, Cryan MJ, Rarity JG, Yu S, O'Brien JL. Silica-on-silicon waveguide quantum circuits. Science. 2008;320:646.

17. Földi P, Kálmán O, Benedict MG, Peeters FM. Quantum rings as electron spin beam splitters. Phys Rev B. 2006:73:155325.

18. Faruque II, Sinclair GF, Bonneau D, Rarity JG, Thompson MG. On-chip quantum interference with heralded photons from two independent micro-ring resonator sources in silicon photonics. Opt Express. 2018;26:646.

19. Kim D, Ibrahim MI, Foy C, Trusheim ME, Han R, Englund DR. A CMOS-integrated quantum sensor based on nitrogen-vacancy centres. Nat Electron. 2019;2:284.

20. Kandala A, Mezzacapo A, Temme K, Takita M, Brink M, Chow JM, Gambetta JM. Hardware-efficient variational quantum eigensolver for small molecules and quantum magnets. Nature. 2017;549:242.

21. Wang J et al. Multidimensional quantum entanglement with large-scale integrated optics. Science. 2018;360:285.

22. Valagiannopoulos C. Steering of quantum signals along coupled paths of arbitrary curvature. J Opt Soc Am B. 2021:38:263.

23. Yu N, Genevet P, Kats MA, Aieta F, Tetienne J-P, Capasso F, Gaburro Z. Light propagation with phase discontinuities: generalized laws of reflection and refraction. Science. 2011;334:333.

24. Asadchy VS, Diaz-Rubio A, Tcvetkova SN, Kwon DH, Elsakka A, Albooyeh M, Tretyakov SA. Flat engineered multichannel reflectors. Phys Rev X. 2017;7:031046.

25. Létoublon A, Favre-Nicolin V, Renevier H, Proietti MG, Monat C, Gendry M, Marty O, Priester C. Strain, size, and composition of InAs quantum sticks embedded in InP determined via grazing incidence $X$-ray anomalous diffraction. Phys Rev Lett. 2004;92:186101.

26. Malachias A, Kycia S, Medeiros-Ribeiro G, Magalhães-Paniago R, Kamins TI, Williams RS. 3D composition of epitaxial nanocrystals by anomalous X-ray diffraction: observation of a Si-rich core in ge domes on Si(100). Phys Rev Lett. 2003;91:176101

27. Christesen JD, Pinion CW, Hill DJ, Kim S, Cahoon JF. Chemically engraving semiconductor nanowires: using three-dimensional nanoscale morphology to encode functionality from the bottom up. J Phys Chem Lett. 2016;7:685.

28. Lauhon L, Gudiksen MS, Wang D, Lieber CM. Epitaxial core-shell and core-multishell nanowire heterostructures. Nature. 2002;470:57.

29. Gazibegovic $S$ et al. Epitaxy of advanced nanowire quantum devices. Nature. 2017;548:434.

30. Hausmann BJM, Khan M, Zhang Y, Babinec TM, Martinick K, McCutcheon M, Hemmerd PR, Loncar M. Fabrication of diamond nanowires for quantum information processing applications. Diam Relat Mater. 2010;19:621.

31. Shirodkar SN, Mattheakis M, Cazeaux P, Narang P, Soljačić M, Kaxiras E. Quantum plasmons with optical-range frequencies in doped few-layer graphene. Phys Rev B. 2018;97:195435.

32. Marton L, Simpson JA, Suddeth JA. Electron beam interferometer. Phys Rev. 1953;90:490.

33. Cronin AD, McMorran B. Electron interferometry with nanogratings. Phys Rev A. 2006;74:061602(R).

34. Gronniger G, Barwick B, Batelaan H. A three-grating electron interferometer. New J Phys. 2006;8:224.

35. Schulz C, Heinisch RL, Fehske H. Electron flow in circular graphene quantum dots. Quantum Matter. 2015;4:346.

36. Gomez-Diaz JS, Tymchenko M, Lee J, Belkin MA, Alù A. Nonlinear processes in multi-quantum-well plasmonic metasurfaces: electromagnetic response, saturation effects, limits, and potentials. Phys Rev B. 2015;92:125429.

37. Liberal I, Ederra I, Ziolkowski RW. Control of a quantum emitter's bandwidth by managing its reactive power. Phys Rev A. 2019;100:023830.

38. Valagiannopoulos C. Perfect quantum cloaking of tilted cylindrical nanocavities. Phys Rev B. 2020;101:195301.

39. Valagiannopoulos C. Optimally sharp energy filtering of quantum particles via homogeneous planar inclusions. Sci Rep. 2020;10:816.

40. Griffiths DJ. Introduction to quantum mechanics. Upper Saddle River: Pearson Prentice Hall; 2005. 
41. Trachanas S. An introduction to quantum physics. New York: Wiley; 2018.

42. Balanis CA. Advanced engineering electromagnetics. New York: Wiley; 1989

43. Stratton JA. Electromagnetic theory. New York: McGraw-Hill; 1941.

44. Abramowitz M, Stegun IA. Handbook of mathematical functions. New York: National Bureau of Standards; 1970.

45. Lewin L. Structural properties of polylogarithms. Mathematical surveys \& monographs. New York; 1991.

46. Tagay Z, Valagiannopoulos C. Highly selective transmission and absorption from metasurfaces of periodically corrugated cylindrical particles. Phys Rev B. 2018;98:115306.

47. Morse PM, Feshbach H. Methods of theoretical physics, part I. International series in pure and applied physics. New York; 1953.

48. Pendry JB. Negative refraction makes a perfect lens. Phys Rev Lett. 2000:85:3966

49. Petit R. Electromagnetic theory of gratings, topics in current physics. vol. 22. Berlin: Springer; 1980.

50. Adachi S. Properties of semiconductor alloys: group-IV, III-V and II-VI semiconductors. New York: Wiley; 2009.

51. Kaxiras E, Joannopoulos JD. Quantum theory of materials. New York: Cambridge University Press; 2019.

52. Beenakker CWJ, Houten HV. Quantum transport in semiconductor nanostructures. Solid State Phys. 1991;44:1-228.

53. Valagiannopoulos CA. Study of an electrically anisotropic cylinder excited magnetically by a straight strip line. Prog Electromagn Res. 2007;73:297-325.

54. Abrashuly A, Valagiannopoulos C. Limits for absorption and scattering by core-shell nanowires in the visible spectrum. Phys Rev Appl. 2019;11:014051.

55. Liu CP, Wang RC, Kuo CL, Liang YH, Chen WY. Recent patents on fabrication of nanowires. Recent Pat Nanotechnol. 2007:1:11

56. Xia X, Tu J, Zhang Y, Wang X, Gu C, Zhao X, Fan HJ. High-quality metal oxide core/shell nanowire arrays on conductive substrates for electrochemical energy storage. ACS Nano. 2012;6:5531.

57. Istrate E, Sargent EH. Photonic crystal heterostructures and interfaces. Rev Mod Phys. 2006;78:455.

58. Nie Z, Petukhova A, Kumacheva E. Properties and emerging applications of self-assembled structures made from inorganic nanoparticles. Nat Nanotechnol. 2010;5:15

59. Brecht T. Micromachined quantum circuits [PhD dissertation]. New Haven: Yale University; 2017.

60. Valagiannopoulos CA. Arbitrary currents on circular cylinder with inhomogeneous cladding and RCS optimization. J Electromagn Waves Appl. 2007;21:665-80.

61. Valagiannopoulos CA. Closed-form solution to the scattering of a skew strip field by metallic pin in a slab. Prog Electromagn Res. 2008;79:1-21.

62. Valagiannopoulos CA. A novel methodology for estimating the permittivity of a specimen rod at low radio frequencies. J Electromagn Waves Appl. 2012;24:631-40.

\section{Submit your manuscript to a SpringerOpen ${ }^{\circ}$ journal and benefit from:}

- Convenient online submission

- Rigorous peer review

- Open access: articles freely available online

- High visibility within the field

- Retaining the copyright to your article

Submit your next manuscript at $\boldsymbol{s p r i n g e r o p e n . c o m ~}$ 